\title{
Localizing the Language Network with fMRI and Functional Connectivity: Implications for Pre-Surgical Planning
}

\author{
Victoria Lyn Ives-Deliperi' ${ }^{1}$ James Thomas Butler ${ }^{1,2}$ \\ ${ }^{1}$ Divison of Neurology, Department of Medicine, University of Cape Town, Cape Town, South Africa \\ ${ }^{2}$ Department of Neurology, University of Stellenbosch, Stellenbosch, South Africa \\ Email: vives@mweb.co.za
}

How to cite this paper: Ives-Deliperi, V.L. and Butler, J.T. (2018) Localizing the Language Network with fMRI and Functional Connectivity: Implications for Pre-Surgical Planning. Open Journal of Modern Neurosurgery, 8, 174-186.

https://doi.org/10.4236/ojmn.2018.82015

Received: January 17, 2018

Accepted: April 8, 2018

Published: April 11, 2018

Copyright (c) 2018 by authors and Scientific Research Publishing Inc. This work is licensed under the Creative Commons Attribution International License (CC BY 4.0). http://creativecommons.org/licenses/by/4.0/

\begin{abstract}
Object: Functional MRI is frequently applied to lateralize language in pre-surgical planning, with potential to localize functionally important cortex too. Here we present BOLD signal activation maps and related functional connectivity, in response to three commonly administered fMRI language tasks. Methods: Datasets from 55 pre-surgical fMRI studies were analyzed. Verbal response naming, covert word generation and passive listening tasks were administered in all studies. Single-subject analyses, group analyses and region-of-interest analyses were conducted, and a multi-subject functional connectivity analysis was performed. Results: Single-subject analyses revealed that clinically important language regions were activated in all but three patients using the panel of tasks. Group analyses revealed significant bilateral BOLD signal increases in anterior and posterior language regions in response to verbal response naming and bilateral signal increase in posterior language regions only in response to passive listening. Covert word generation activated anterior language regions bilaterally and posterior language cortex in the dominant hemisphere. Functional connectivity analyses confirmed that activated regions were significantly correlated in all tasks. Conclusion: The findings of single-subject and group analyses add to the evidence supporting the use of a panel of fMRI tasks to map the language network for pre-surgical planning. Our findings support the additional use of functional connectivity analysis in routine language mapping to add to the localization value to fMRI. In addition, the results of our investigation demonstrate these three commonly applied tasks reliably activate unique aspects of the language network, which advocates closer individual inspection, guided by the surgical intervention planned.
\end{abstract}




\section{Keywords}

Brain Mapping, fMRI, Functional Connectivity, Language, Pre-Surgical Planning

\section{Introduction}

Neurosurgical intervention carriers the risk of adverse effects on cognitive and motor functioning. Localizing eloquent cortex ensures that functionally important brain regions are protected during surgery, while maximizing the area of resection for optimal outcome. Language is one of the most common functions to be mapped in neurosurgical planning.

fMRI is considered a reliable tool to identify the language-dominant hemisphere, with potential localization value too [1]. Lateralization of language by fMRI and Wada has been shown to be concordant in up to $86 \%$ of individuals [2] [3]. Although direct cortical stimulation mapping (DCS) remains the gold standard of localizing language cortex, novel approaches to improving the localization value of fMRI are encouraged to either expedite DCS or standalone when DCS is unfeasible or unsuccessful [4].

It is recommended that a panel of tasks be administered in fMRI language [5]. The aim of this study was to more closely inspect the variable activation patterns generated by three commonly administered tasks and the functional connectivity between activated regions for the benefit of clinicians tasked with pre-surgical language mapping and of interest to neurosurgeons planning resections in the dominant hemisphere. The findings are aimed to inform the development of methods to localize specific language regions for surgical planning.

\section{Materials and Methods}

Functional MRI datasets for patients undergoing surgical resections from 2011 to 2016 were retrieved from a single neurosurgical unit. A possible 256 data sets were identified of which $55 \mathrm{fMRI}$ studies included the administration of all three language tasks used for surgical planning in the unit, including verbal response naming, covert word generation and passive listening. Neither ethics approval nor patient informed consent was required for this retrospective study, as outlined by the National Code for Clinical Trials, as all data analyzed were collected as part of routine diagnosis and treatment.

\section{1. fMRI Data Acquisition}

Scans were acquired using a 1.5 T Avanto MRI scanner (Siemens Medical Systems, Erlangen, Germany). High-resolution anatomical images were acquired in the sagittal plane using a three-dimensional inversion recovery gradient echo sequence (160 slices, $1.2-\mathrm{mm}$ thick. Skip $=0 ; \mathrm{TR}=2400 \mathrm{~ms}$; TE $=3.61 \mathrm{~ms}$; flip 
angle $=8^{\circ}$; field of view $=240 \times 240$; voxel size $=1 \times 1 \times 1 \mathrm{~mm}$; matrix size $=$ $192 \times 192$ ). During the fMRI protocol 110 functional volumes sensitive to blood oxygen level dependent contrast were acquired in all mapping tasks with a $\mathrm{T} 2 *$-weighted gradient echo, echo planar imaging sequence $(\mathrm{TR}=2000 \mathrm{~ms}, \mathrm{TE}=$ $50 \mathrm{~ms}, 21$ interleaved slices, $5 \mathrm{~mm}$ thick, gap $1.25 \mathrm{~mm}$, matrix size $64 \times 64$, resolution $3.4 \times 3.4 \times 3.4 \mathrm{~mm}^{3}$ ).

\subsection{Scanning Protocol}

The scan commenced with a 6-minute T1 sequence to generate structural data, followed by the three functional paradigms. Each task consisted of 10 alternating blocks of $20 \mathrm{~s}$ commencing with $20 \mathrm{~s}$ rest (total = 3:66 min). In all language tasks, stimuli in the control condition consisted of high and low tones to engage auditory processing and attention. Participants were instructed to listen to the tones attentively. In the active condition of the tasks stimuli were projected on a screen at the base of the bed and viewed through an overhead mirror in the head coil. Foam padding was used around the head to reduce motion.

In the active conditions of the tasks patients were asked to a) silently name objects being described by short sentence presented aurally and visually in verbal response naming, b) silently generate verbs associated with nouns presented aurally and visually in word generation, and c) listen to a story presented aurally in passive listening.

\section{3. fMRI Data Analyses}

All fMRI analyses were performed using Brain Voyager QX (Brain Innovation, Maastricht, The Netherlands). Two dummy images were excluded from analysis in each task run. Images were motion corrected relative to the first volume with trilinear estimation and interpolation. Images were corrected for different slice acquisition times and linear trends, spatially smoothed using a Gaussian filter (FWHM $4 \mathrm{~mm}$ ), and temporally smoothed with a high pass filter of 3 cycles/ point. Data sets were rejected if they exceeded movement criteria $3 \mathrm{~mm}$ displacement, 3.0 rotation within a functional run. Each subject's functional data was co-registered to 1) his/her high-resolution native anatomical MRI for single-subject analyses, and 2) to their anatomical MRI normalized in Talairach space, rotated into the AC-PC plane, for the benefit of group analyses. The $3.4 \times$ $3.4 \times 3.4 \mathrm{~mm}^{3} \mathrm{fMRI}$ voxels were interpolated during Talairach normalization to $3 \times 3 \times 3 \mathrm{~mm}^{3}$.

\subsubsection{Whole-Brain Single-Subject and Group Analyses}

For each task, a fixed-effect analysis of variance was performed using the general linear model (GLM) with one predictor for the active conditions convolved by the standard hemodynamic function. BOLD signal changes were examined by comparing active and control conditions. The voxel-wise threshold was set to $p$ $<0.01$ (voxel-wise Bonferroni corrected for whole-brain multiple comparisons, $\min t$ statistic 7.6). Clusters were reported if their extent was greater than five 
contiguous voxels, where the voxel size refers to the $1 \times 1 \times 1 \mathrm{~mm}^{3}$ resolution of the iso-voxeled structural images.

\subsubsection{Functional Connectivity Analyses}

A region-of-interest analysis was performed on the group activation maps generated by each task. The average beta-values in the time-series of all patients in all activated regions during task performance were extracted and entered into a spreadsheet. Beta-values were averaged and correlation analyses were performed on the averaged values in each language region. In addition to this, a subgroup analysis was conducted to investigate potential differences in functional connectivity between patients with left TLE, compared to right TLE.

\subsubsection{Language Lateralization}

Lateralization indices (LI) were calculated in single-subject and group activation map results for each task. All the active voxels in the left hemisphere were subtracted from active voxels in the right hemisphere, divided by the active voxels in the left hemisphere added to the active voxels in the right hemisphere, with cut-off values of $0.2 /-0.2$ used to confirm dominance [6]. Lateralization indices were also calculated from the BOLD activations maps generated in multi-subject analyses of each task and referred to here as group LIs.

\section{Results}

The initial patient cohort consisted of 55 pre-surgical patients, 24 males and 31 females, ranging from 20 to 54 years (mean age \pm standard deviation [SD], 35.6 \pm 13 years). Right-hand dominance was confirmed in 52 patients, two were left-hand dominant and one was mixed-handed. Seizure disorders were present in all but three patients, the latter of whom were awaiting left-hemisphere tumor resections. Of the patients with seizures disorders, 34 were diagnosed with TLE, 22 of whom had dominant hemisphere TLE and 12 in which hippocampal sclerosis was identified on MRI. Nine patients were diagnosed with frontal lobe epilepsy, in which electrographic disturbances in the dominant hemisphere were present in six, five were diagnosed with occipital lobe epilepsy, four of which showed dominant hemisphere abnormalities on EEG, and finally four with epileptogenic discharges over cortical dysplasias in the dominant hemisphere. Tumors in three of the patients were close to, but not directly infringing upon language cortex and there were no other patients with mass lesions. All patients with epilepsy were deemed suitable for surgery following long-term video-EEG monitoring, and had fMRI as part of the pre-surgical work-up. All patients were cooperative and able to perform the fMRI tasks.

Single-subject analyses were conducted in all patients for the purposes of pre-surgical planning. Significant BOLD signal increases were generated in predefined important language regions, including Broca's area, Exner's area, supplementary speech area/SMA, Wernicke's area, angular gyrus and basal temporal language cortex, in all but three patients. In addition, one patient was 
right-hemisphere dominant for language. These four patients were excluded from further group analyses.

\subsection{Group Analyses}

\subsubsection{Verbal Response Naming}

The BOLD signal activation map resulting from the group analysis of 51 patients who underwent verbal response naming revealed significant BOLD signal increases in frontal and temporal lobe language centers involved in expressive and receptive language functions (Figure 1). The regions included the posterior, inferior aspect of the frontal convexity (BA44/Broca's area) in the left hemisphere $t(47)=7.35, \mathrm{p}=0.001$ and right hemisphere $t(47)=6.39, \mathrm{p}=0.001$, the posterior middle frontal gyrus in the left hemisphere (Exner's area) $t(47)=6.22, \mathrm{p}=0.001$, the supplementary speech area (sensory motor area SMA; BA6) $t(47)=7.46, \mathrm{p}=$ 0.001 and the posterior part of the superior temporal gyri (BA22/Wernicke's area) bilaterally $(t(47)=6.36, \mathrm{p}=0.001$ and $t(47)=6.95, \mathrm{p}=0.001)($ Table 1$)$. More intense and wider-spread activations were noted in the left hemisphere and while all the LIs in single-subjects analyses were above 0.1 , only 42 of the 51 patients met the criteria for left-hemisphere dominance ( $L I>/=0.2)$. The lateralization index for the group fell below the cut-off at 0.18 . Significant signal decreases were also noted in the precuneus (BA 7) $t(47)=-8.21, \mathrm{p}=0.001$. Although the task generated signal activation in the basal temporal region of the left hemisphere, this was only evident slightly below the threshold of significance $(\mathrm{p}=0.0503)$.

\subsubsection{Covert Word Generation}

Activation maps generated for the group analysis of the covert word generation task overlapped that of verbal response naming, but only in the dominant hemisphere for receptive language function (Figure 1). Significant BOLD signal increases were evident in anterior language cortex bilaterally, left; $t(47)=9.29, \mathrm{p}=$ 0.001 and right; $t(47)=6.16, \mathrm{p}=0.001$, and in the posterior language region in the left hemisphere $t(47)=5.48, \mathrm{p}=0.001$. Significant signal increase was also noted in SMA $t(47)=5.84, \mathrm{p}=0.001$, and decreases in the precuneus $t(47)=$ $-9.46, \mathrm{p}=0.001$ (Table 1). The LIs were greater than 0.2 in all but one patient and the group LI was 0.59 .

\subsubsection{Passive Listening}

The group analysis of passive listening showed significant BOLD signal increases in the posterior language areas bilaterally $t(47)=7.18, \mathrm{p}=0.001$ and $t(47)=$ $6.56, \mathrm{p}=0.001$, and significant signal decreases in precuneus $t(47)=-5.23, \mathrm{p}=$ 0.001 and $t(47)=-5.21, \mathrm{p}=0.001$. No signal changes were generated in anterior language regions in the frontal lobe (Figure 1 and Table 1). Comparable with findings from the verbal response task, LIs were equally to or great than greater than 0.1 in all patients but greater than 0.2 in 40 of the 51 patients. The group LI was 0.15 . 

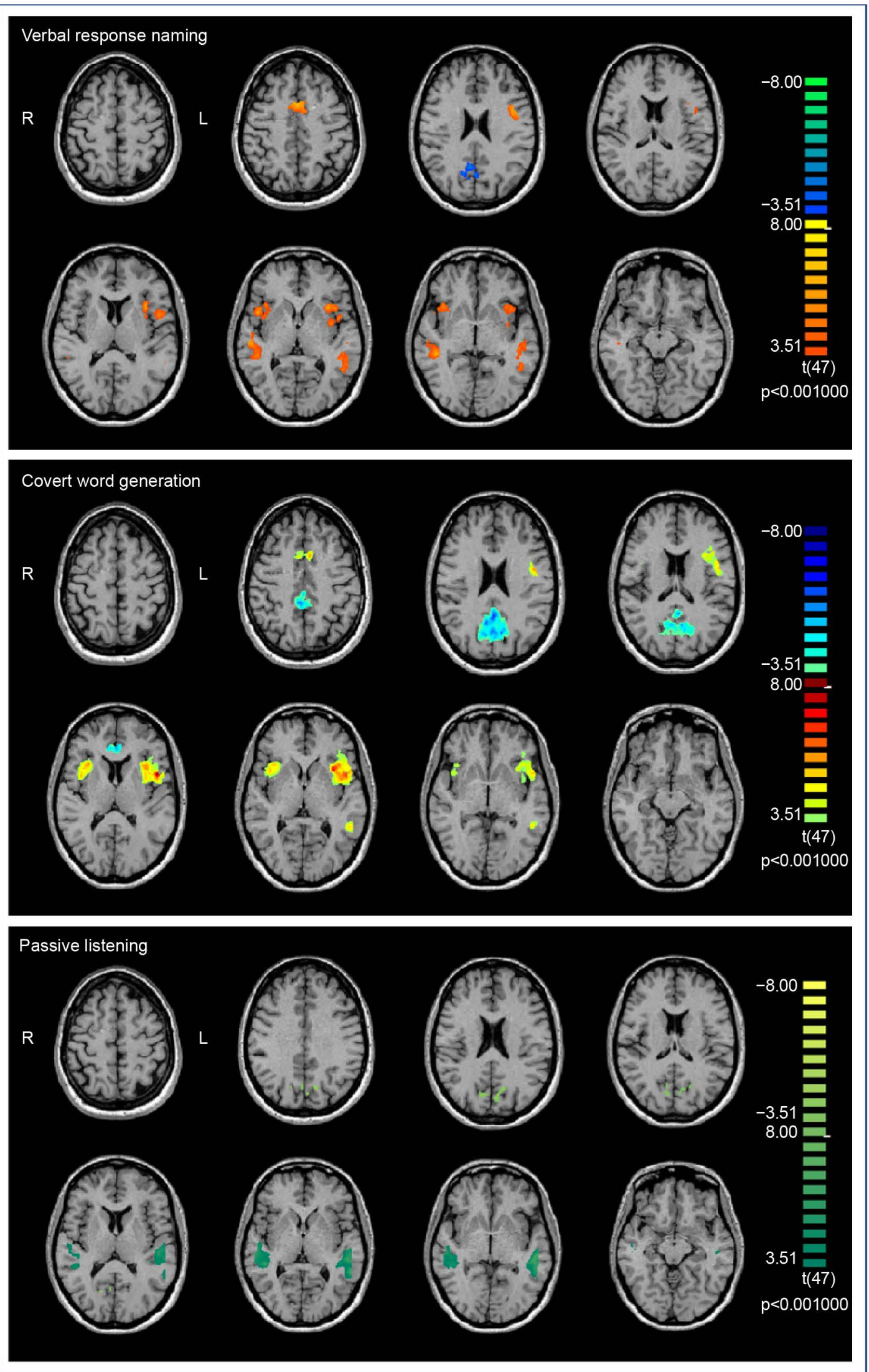

Figure 1. Whole brain group analyses of language tasks. 
Table 1. Functional MRI BOLD signal changes.

\begin{tabular}{|c|c|c|c|c|c|c|}
\hline \multirow[b]{2}{*}{ Brain region } & \multirow[b]{2}{*}{ BA } & \multicolumn{5}{|c|}{ Talairach co-ordinates } \\
\hline & & Voxels & Peak $\mathbf{x}$ & Peak y & Peak z & Voxel T \\
\hline \multicolumn{7}{|l|}{ Verbal response naming } \\
\hline LH Broca's area & BA 44 & 3853 & -48 & 6 & 11 & 7.35 \\
\hline RH Broca's area & BA 44 & 2766 & 45 & 11 & 3 & 6.39 \\
\hline LH Exner's area & BA 6 & 2104 & -40 & 2 & 27 & 6.22 \\
\hline LH Wernicke's area & BA 22 & 2932 & -48 & -28 & -3 & 6.36 \\
\hline RH Wernicke's area & BA 22 & 3306 & 45 & -34 & -3 & 6.95 \\
\hline Supplementary speech area & BA 6 & 3370 & 0 & 9 & 43 & 7.46 \\
\hline LH & & 1942 & -3 & 9 & 43 & 5.67 \\
\hline RH & & 1428 & 4 & 11 & 41 & 5.31 \\
\hline Precuneus & BA 7 & 4141 & 3 & -58 & 27 & -8.21 \\
\hline $\mathrm{LH}$ & & 278 & -9 & -63 & 22 & -4.38 \\
\hline RH & & 3863 & 3 & -58 & 27 & -5.66 \\
\hline \multicolumn{7}{|l|}{ Covert word generation } \\
\hline LH Broca's area & BA 44 & 12071 & -48 & 8 & 9 & 9.29 \\
\hline RH Broca's area & BA 44 & 3119 & 33 & 17 & 6 & 6.16 \\
\hline LH Wernicke's area & BA 22 & 656 & -51 & -46 & 0 & 5.48 \\
\hline Supplementary speech area & BA 6 & 584 & -6 & 11 & 42 & 5.85 \\
\hline $\mathrm{LH}$ & & 301 & -6 & 11 & 42 & 3.43 \\
\hline RH & & 283 & 4 & 10 & 42 & 2.75 \\
\hline Precuneus & BA 7 & 19094 & -6 & -46 & 36 & -9.46 \\
\hline $\mathrm{LH}$ & & 9942 & -6 & -46 & 36 & -6.70 \\
\hline $\mathrm{RH}$ & & 9152 & 8 & -57 & 27 & -6.13 \\
\hline \multicolumn{7}{|l|}{ Passive listening } \\
\hline LH Wernicke's area & BA 22 & 5701 & -54 & -25 & -3 & 7.18 \\
\hline RH Wernicke's area & BA 22 & 4201 & 45 & -37 & 3 & 6.57 \\
\hline LH Precuneus & BA 7 & 1283 & -6 & -76 & 24 & -5.23 \\
\hline RH Precuneus & BA 7 & 1190 & 18 & -67 & 12 & -5.21 \\
\hline
\end{tabular}

Note: LH, left hemisphere; RH, right hemisphere; BA, Brodmann Area.

\subsection{Functional Connectivity Analysis}

Region of interest (ROI) analyses were conducted to further interrogate the group activation maps associated with each of the three language tasks.

Brain regions activated in response to verbal response naming shared the more complex array of connections. There was a significantly positive correlation between the Broca's Areas in the two hemispheres $(r=0.94, \mathrm{p}<0.001)$ and between activations in Broca's area in the left hemisphere and Exner's area $(r=$ 
$0.96, \mathrm{p}<0.001)$ and Wernicke's area in the ipsilateral hemisphere $(r=0.90, \mathrm{p}<$ $0.001)$, Wernicke's area in the contralateral hemisphere $(r=0.90, \mathrm{p}<0.001)$, SMA $(r=0.93, \mathrm{p}<0.001)$ and precuneus $(r=0.23, \mathrm{p}<0.05)$. There was a highly significant correlation between Exner's area and Wernicke's area in the ipsilateral hemisphere $(r=0.89, \mathrm{p}<0.001)$. Significant positive correlations were also evident between activations in Broca's area in the right hemisphere and Wernicke's areas in the left hemisphere $(r=0.81, \mathrm{p}<0.001)$ and right hemisphere $(r$ $=0.83, \mathrm{p}<0.001)$, and SMA $(r=0.86, \mathrm{p}<0.001)$ and precuneus $(r=0.38, \mathrm{p}<$ $0.001)$. Significantly positive correlations were also evident between activations in Wernicke's areas in the two hemispheres $(r=0.95, \mathrm{p}<0.001)$ and Wernicke's area in the left hemisphere and SMA $(r=0.89, \mathrm{p}<0.001)$. Activations in Wernicke's area in the right hemisphere was significantly correlated with those in SMA $(r=0.92, \mathrm{p}<0.001)$.

In response to covert word generation, activations in Broca's area in the left hemisphere were significantly correlated with those in the right hemisphere $(r=$ $0.95, \mathrm{p}<0.001)$, Wernicke's area in the ipsilateral hemisphere $(r=0.90, \mathrm{p}<$ $0.001)$ and SMA $(r=0.93, \mathrm{p}<0.001)$. Activations in Broca's area in the right hemisphere was also strongly correlated with activation in Wernicke's area in the left hemisphere $(r=0.86, \mathrm{p}<0.001)$ and SMA $(r=0.90, \mathrm{p}<0.001)$. Significant correlations were also evident between activations in Wernicke's area in the left hemisphere and SMA $(r=0.88, \mathrm{p}<0.001)$.

Activations in Wernicke's area in the left and right hemispheres in response to passive listening were significantly correlated $(r=0.96, \mathrm{p}<0.001)$ and activations in Wernicke's area in the left hemisphere were significantly correlated with those in the left and right precuneus (LH: $r=0.20, \mathrm{p}<0.05$ and $\mathrm{RH}:(r=0.27, \mathrm{p}$ $<0.01)$. Bilateral precuneus activation were also highly correlated $(r=0.91, \mathrm{p}<$ 0.001). These results are graphically depicted in Figure 2.

According to the subgroup analyses, there was no significant difference in connectivity within the language network between patients with dominant TLE and non-dominant TLE, although there was a trend towards weaker correlations between activated regions patients with left TLE.

\section{Discussion}

The purpose of this study was to investigate variable activations in the language network, generated by three commonly administered fMRI language tasks and the implications for pre-surgical planning. The findings show 1) verbal response naming generates widespread, bilateral BOLD signal increases in the language network, 2) covert word generation produces the most discrete activations in anterior and posterior language regions in the dominant hemisphere, with the most impressive lateralization value of the three tasks and 3) passive listening targets receptive language regions bilaterally. BOLD activation maps generated by the three individual tasks were consistent with reported findings from previous studies [4] [7] [8]. The investigations also show that 4) connectivity 


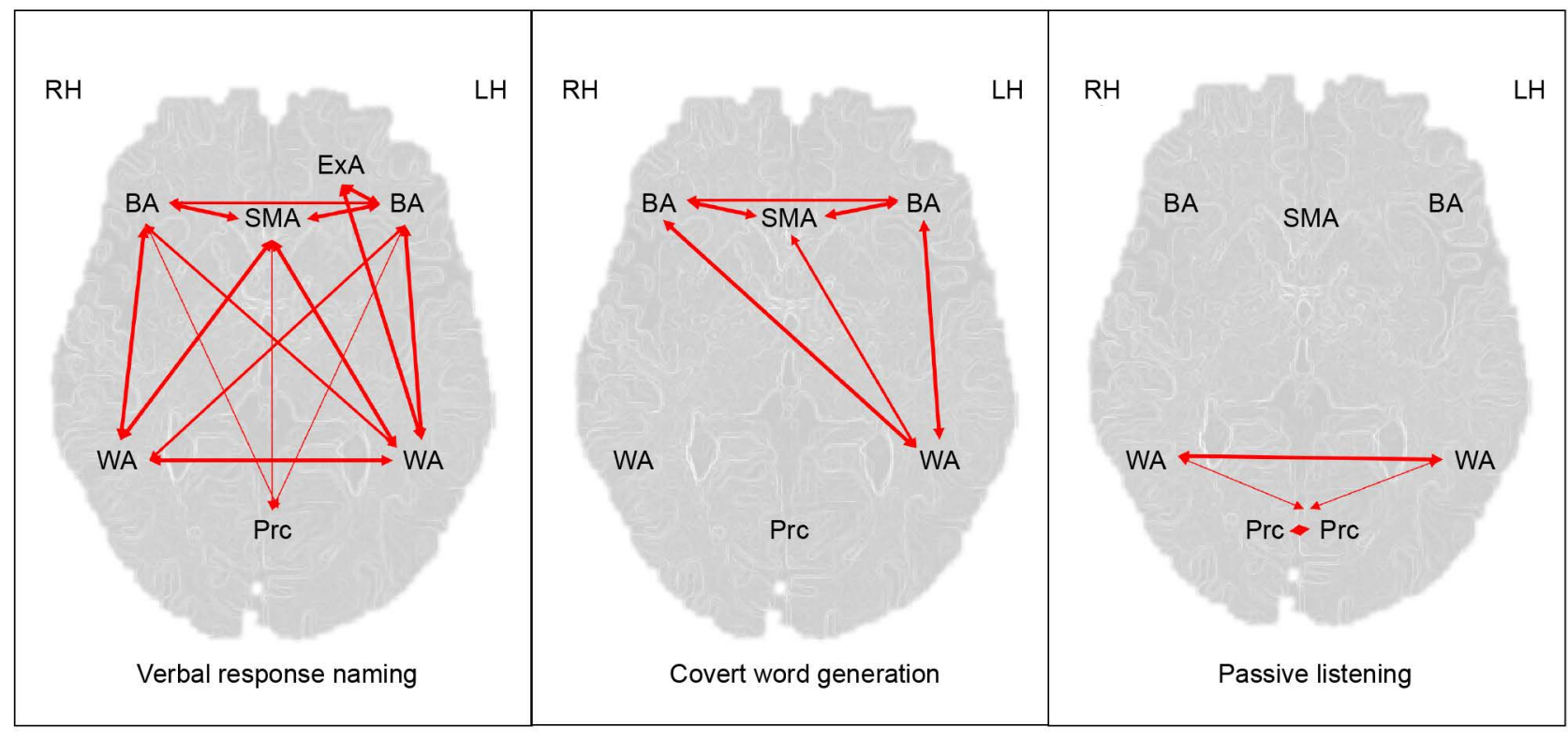

$\longleftrightarrow$ Positive correlation at $p<0.001 \longleftrightarrow$ Positive correlation at $p<0.01$

Figure 2. Functional connectivity between activated regions in the three language tasks.

between the language regions showing significant BOLD signal increases in all three tasks are functionally connected, which supports the notion that the regions subserve language and 5) there were no significant differences in connectivity between language regions in patients with dominant TLE and non-dominant TLE.

\subsection{Language Lateralization}

Determining hemispheric laterality may be achieved by either calculating a LI or visual inspection of fMRI activation maps by experienced clinicians [4]. Language lateralization was deemed satisfactory by visual inspection in all 51 single-subject analyses in this study, across all tasks. Hemispheric dominance was confirmed by calculating LIs in $80 \%$ of the single-subject activation maps generated by the verbal response naming and passive listening tasks, and in $100 \%$ of related activation maps generated by covert word generation. Verbal response naming and passive listening generate bilateral signal increases, however, activations in the left hemisphere are more intense and wider spread than in the right hemisphere. Right hemisphere signal activations in anterior and posterior language cortex were more striking in the group analyses than in single-subject analyses, and consistent with findings from resting state connectivity analyses [9].

\subsection{Language Localization}

\subsubsection{Anterior Language Regions}

Broca's area, and occasionally Exner's areas, are primary targets when localizing expressive language regions in the frontal lobe during DCS. Localizing these areas on anatomical morphometry alone is unreliable, particularly in patients with space-occupying lesions [10]. In the findings of the group analyses in this study, 
verbal response naming and covert word generation reliably activated Broca's area but only the former task engaged Exner's areas. In four out of five patients who underwent DCS in our cohort, speech arrest occurred in a congruent location to fMRI activations in Broca's area. While Exner's area is seldom a target for localization during DCS at our center, stimulation of the region in the DLPFC has shown to temporarily disrupt handwriting [11]. Verbal response naming and covert word generation both produced reliable activation in supplementary speech area (SMA), bilaterally in this investigation. This region is involved in initiating and sequencing of motor movements for speech and the extent of its involvement in language is highly variable between patients [8]. Although unilateral resections may produce transient aphasia, the condition recovers postoperatively and thus SMA cannot strictly be considered a critical language area [12] [13]. fMRI activations in this region are potentially informative nonetheless, in that the presence of BOLD signal changes in the contralateral healthy SMA and diminished BOLD signal in the ipsilateral SMA in patients with low grade glial tumours have been shown to correlate with the speed of recovery from the post-operative SMA syndrome [12].

\subsubsection{Posterior Language Regions}

The localization of critical receptive language regions in the temporal lobe is more challenging, both in fMRI and DCS. Primary areas targeted in mapping of receptive language include Wernicke's' area, Angular gyrus and basal temporal regions. DCS stimulation in the region of angular gyrus has been shown to interrupt handwriting and repetition and disrupt naming, comprehension, reading and repetition when applied to more basal regions of the temporal lobe, fusiform and parahippocampal gyri [4] [13].

Verbal response naming and covert word generation involve reading, comprehension of the written sentence and naming and thus, predictably, activate the posterior language regions demonstrated in this investigation. Verbal response naming was the only task in the panel of three to activate the basal temporal language region, according to groups analyses, possibly related to the increased reading load of the task. Although the covert word generation task produced signal activations in basal temporal region below the threshold of significant, activations in the regions were noted in a number of single-subject analyses in response to the task. Surgical resection of the basal temporal region, however, seldom produces lasting language deficits so, like SMA, may be considered a secondary language area [14]. The findings from the analyses of the passive listening task were helpful in this investigation, in so far as that the location of signal activations corroborated findings from the other tasks, and produced the most intense and widest activation in Wernicke's area.

DCS of posterior language regions is less reliable compared to stimulation in anterior regions. As opposed to speech arrest, stimulation can elicit comprehension difficulties, anomia and paraphasic errors. In our experience, extra-operative and intra-operative ECS elicits relatively subtle dysfunction, which brings into 
question how best critical language cortex in the temporal lobe may be systematically mapped. This warrants further investigation. The margins of craniotomies exposed the regions of temporal lobe activated in the fMRI tasks in only two of the five patients in our cohort who underwent awake speech mapping. Anomia was elicited in one of these patients when Wernicke's area was stimulated.

BOLD signal decreases were evident in the precuneus in response to all three language tasks in this study, consistent with previous findings of reduced activity in the region during the performance of a variety of higher-order cognitive functions [15].

\subsection{Functional Connectivity of Language Regions}

Studies have shown reduced functional connectivity between language regions in patient with TLE compared to healthy subjects [16] [17]. This is likely due to intra-hemispheric and interhemispheric language reorganization in localized epilepsy [18]. Recent studies have also demonstrated reduced connectivity in the language network between patients with dominant and non-dominant TLE [9]. No significant differences in connectivity between the groups was evident in this study, perhaps due to our larger cohort.

\section{Conclusions}

The findings of this investigation are informative in so far as they may guide fMRI task selection and analysis in pre-surgical fMRI language mapping, guided by the proposed area for resection. Since verbal response naming generates widespread, bilateral BOLD signal increases in the language network, this task would be the most useful when there is a requirement to map the entire language network, for example in speech and language research. Should language lateralization be the primary requirement, then the task of choice would be covert word generation, which produces the most discrete activations in anterior and posterior language regions in the dominant hemisphere, with the most impressive lateralization value of the three tasks. It would be particularly useful to include a passive listening task when attempting to localize posterior language regions in the case of temporal lobe resections. The addition of functional connectivity analyses in fMRI studies helps to further corroborate the assumptions made about localization of eloquent cortex from task-based fMRI findings.

This study was limited by the relatively heterogeneous population and investigating BOLD signal activations in response to these tasks in a healthy cohort would be of interest. Significantly weaker connectivity between language regions were notable in the three tumor patients compared to the group as a whole and it would be recommended to explore such connectivity in a larger sample of tumor patients. To confirm the clinical usefulness of the tasks studied in this research, BOLD signal activation patterns need to be measured against DCS results and post-operative language outcomes. The addition adding DTI to imaging 
protocol for the mapping white matter tracts involved in language, as well as DCS of these tracts, is also strongly recommended for the preservation of function [19].

\section{Disclosures}

The authors report no conflict of interest concerning the materials or methods used in this study or the findings specified in this paper.

\section{References}

[1] Szaflarski, J.P., Binder, J.R., Gaillard, W.D., Golby, A.J., Holland, S.K., Ojemann, J., et al. (2017) Practice Guideline Summary: Use of fMRI in the Presurgical Evaluation of Patients with Epilepsy. Report of the Guideline Development, Dissemination, and Implementation Subcommittee of the American Academy of Neurology. Neurology, 88, 395-402. https://doi.org/10.1212/WNL.0000000000003532

[2] Abbott, D.F., Waites, A.B., Lillywhite, L.M. and Jackson, G.D. (2010) fMRI Assessment of Language Lateralization: An Objective Approach. Neuroimage, 50, 1446-1455. https://doi.org/10.1016/j.neuroimage.2010.01.059

[3] Janecek, J.K., Swanson, S.J., Sabsevitz, D.S., Hammeke, T.A., Raghavan, M., Rozman, M., et al. (2013) Language Lateralization by fMRI and Wada Testing in 229 Patients with Epilepsy: Rates and Predictors of Discordance. Epilepsia, 54, 314-322. https://doi.org/10.1111/epi.12068

[4] Benjamin, C.F., Walshaw, P.D., Hale, K., Gaillard, W.D., Baxter, L.C., Berl, M.M., et al. (2017) Presurgical Language fMRI: Mapping of Six Critical Regions. Human Brain Mapping, 38, 4239-4255. https://doi.org/10.1002/hbm.23661

[5] Duncan, J.S. (2010) Imaging in the Surgical Treatment of Epilepsy. Nature Review Neurology, 6, 537-550. https://doi.org/10.1038/nrneurol.2010.131

[6] Seghier, M.L. (2008) Laterality Index in Functional MRI: Methodological Issues. Magnetic Resonance Imaging, 26, 594-601.

https://doi.org/10.1016/j.mri.2007.10.010

[7] Smits, M., Visch-Brink, E., and Schraa-Tam, C.K. (2006) Functional MR Imaging of Language Processing: An Overview of Easy-to-Implement Paradigms for Patient Care and Clinical Research. RadioGraphics, 26, 145-159. https://doi.org/10.1148/rg.26si065507

[8] Brennan, N.P., Peck, K.K. and Holodny, A. (2016) Language Mapping Using fMRI and Direct Cortical Stimulation for Brain Tumor Surgery: The Good, the Bad, and the Questionable. Topics in Magnetic Resonance Imaging, 25, 1-10.

https://doi.org/10.1097/RMR.0000000000000074

[9] Pravata, E., Sestieri, C., Mantini, D., Briganti, C., Colicchio, G., Marra, C., et al. (2011) Functional Connectivity MR Imaging of the Language Network in Patients with Drug-Resistant Epilepsy. American Journal of Neuroradiology, 32, 532-570. https://doi.org/10.3174/ajnr.A2311

[10] Keller, S.S., Crow, T., Foundas, A., Amunts, K. and Roberts, N. (2009) Broca's Area: Nomenclature, Anatomy, Typology and Asymmetry. Brain Language, 109, 29-48. https://doi.org/10.1016/j.bandl.2008.11.005

[11] Roux, F.E., Dufor, O., Giussani, C., Wamain, Y., Draper, L., Longcamp, M. and Demonet, J.F. (2009) The Graphemic/Motor Frontal Area Exner's Area Revisited. Annuls of Neurology, 66, 537-545. https://doi.org/10.1002/ana.21804 
[12] Krainik, A., Lehericy, S., Duffau, H., Capelle, L., Chainay, H., Cornu, P., et al. (2003) Postoperative Speech Disorder after Medial Frontal Surgery: Role of the Supplementary Motor Area. Neurology, 60, 587-594. https://doi.org/10.1212/01.WNL.0000048206.07837.59

[13] Binder, J.R. (2015) The Wernicke Area. Neurology, 85, 2170-2175. https://doi.org/10.1212/WNL.0000000000002219

[14] Lüders, H., Lesser, R.P., Hahn, J., Dinner, D.S., Morris, H.H., Wyllie, E., et al. (1991) Basal Temporal Language Area. Brain, 114, 743-754. https://doi.org/10.1093/brain/114.2.743

[15] Shulman, G.L., Fiez, J.A., Corbetta, M., Buckner, R.L., Meizin, F.M., Raichle, M.E., et al. (1997) Common Blood Flow Changes across Visual Tasks: II. Decreases in Cerebral Cortex. Journal of Cognitive Neuroscience, 9, 648-663.

https://doi.org/10.1162/jocn.1997.9.5.648

[16] Kucukboyaci, N.E., Kemmotsu, N., Cheng, C.E., Girard, H.M., Tecoma, E.S., Iragui, V.J., et al. (2013) Functional Connectivity of the Hippocampus in Temporal Lobe Epilepsy: Feasibility of a Task-Regressed Seed-Based Approach. Brain Connectivity, 3, 464-474. https://doi.org/10.1089/brain.2013.0150

[17] Maccotta, L., He, B.J., Snyder, A.Z., Eisenman, L.N., Benzinger, T.L., Ances, B.M., et al. (2013) Impaired and Facilitated Functional Networks in Temporal Lobe Epilepsy. Neuroimage: Clinical, 2, 862-872. https://doi.org/10.1016/j.nicl.2013.06.011

[18] Gaillard, W.D., Berl, M.M., Moore, E.N., Ritzl, E.K., Rosenberger, L.R., Weinstein, S.L., et al. (2007) Atypical Language in Lesional and Nonlesional Complex Partial Epilepsy. Neurology, 69, 1761-1771. https://doi.org/10.1212/01.wnl.0000289650.48830.1a

[19] Duffau, H. (2015) Stimulation Mapping of White Matter Tracts to Study Brain Functional Connectivity. Nature Review Neurology, 11, 255-265. 